\title{
LA PROSTITUCIÓN EN LA HABANA EN LOS PRIMEROS AÑOS DEL SIGLO XX
}

\author{
Alberto J. Gullón Abao \\ Universidad de Cádiz
}

\section{RESUMEN}

Con este trabajo hemos tratado de vislumbrar los cambios que se produjeron en la prostitución reglamentada de la ciudad de La Habana tras la ocupación norteamericana. Para ello hemos analizado, las nuevas propuestas reglamentarista, centrando el estudio en los informes médicos de la época y analizando las modificaciones que, a nuestro juicio, nos han parecido más representativas.

Palabras clave:Cuba, s.XIX. Prostitución.

\section{ABSTRACT}

With this work we have tried to view the changes which occurred at the prostitution of La Habana after the U.S. occupation. In order to offer this analysis we have delt with the "reglamentarist" suggestions at the beginning of the $20^{\text {th }}$. Century. The analysis itself is centred on the medical researches of this period and the most representative changes these researches took to.

Keywords: Cuba, XIXth century. Prostitución

\section{Introducción}

Con este trabajo continuamos una línea abierta hace algunos años sobre el estudio de la prostitución en Cuba. Pretendemos un acercamiento inicial a este período republicano, donde esperamos vislumbrar, si los hubo, los cambios que se produjeron en el sistema reglamentista que predominó durante el final de la colonia, las transformaciones que se experimentaron en la organización médicopolítica interna, los posibles cambios que se generaron sobre la definición de meretriz y el trato a dar a estas mujeres, la efectividad del nuevo sistema, los nuevos problemas que surgen a raíz de la aparición de las nuevas leyes, etc...

Hay que tener presente que nos encontramos en una primera fase de estudio y nos falta revisar documentación (informes policiales, pleitos, censos sanitarios, 
cartillas sanitarias, etc..) que de algún modo nos permita, aunque sean indirectamente, escuchar las voces de estas mujeres. Como fuentes documentales para desvelar los posibles cambios que se produjeron en estos años, analizaremos los primeros reglamentos del Servicio de Higiene Pública y los memoriales de diversos médicos que ocuparon algún cargo en el Servicio. Estos escritos son de una gran riqueza al estar elaboradas por personajes con un amplio conocimiento del tema, pero hay que someterlas a un riguroso análisis ante la posibilidad de transmitir los valores específicos de unos grupos dominantes con sus prejuicios y limitaciones.

\section{La nueva reglamentación}

“..., pues siempre la prostitución ha sido materia dificil de tratar por los recelos y las suspicacias que en unos despierta y la repugnancia que a otros causa, sin tener en cuenta que los sujetos que son objetos de estudio son unos seres desgraciados, dignos de toda clase de consideraciones por la miserable y dificil situación en que se han colocado y merecedores de que se hagan todos los esfuerzos posibles para mejorar su estado buscando al mismo tiempo la mejor manera de garantizar la salud pública."'

Sin caer en el juicio miserabilista, muy extendido en los informes de la época y aún hoy día ${ }^{2}$, destacariamos de la cita anterior dos factores. El primero, que suscribiríamos también para la actualidad, sería que la prostitución era un tema complejo de tratar por los tabúes y prejuicios de la sociedad dominante del momento, ya que ponía de manifiesto ciertos problemas que se pretendían ocultar o difuminar, y el segundo, sería la preocupación sanitaria existente desde el $\mathrm{S}$. XVIII por el tema de enfermedades venéreas que afectan a la salud pública y que facilitó la aparición de reglamentos cuyo objetivo era controlar y eliminar los males venéreos.

Sabemos por los documentos consultados que el primer reglamento con secciones específicas que se aprobó en Cuba fue en 1873, aunque hubo intentos más tempranos en la Isla ${ }^{3}$. Estos reglamentos fueron modificados en función de las necesidades que iban surgiendo y ante la ineficacia para luchar contra las enfer-

\footnotetext{
${ }^{1}$ MOLINET, Eugenio: Memoria informe de la Sección Médica de la Higiene Especial correspondiente al año de 1988. Habana. Imprenta de Francisco Xiqués 1900.

"VÁZQUEZ, Francisco: "Historia de la prostiturción. Problemas metodológicos y niveles del fenómeno. Fuentes y modelos de análisis". En VÁZQUEZ, Francisco (coord): Mal Menor, Políicas y representaciones de la prostitución. Siglos XVI-XLX. Cádiz, 1998.

'Proposición de D. Fernando de Escobar, doctor en Medicina y Cirugía y profesor público de esta ciudad, al Gobernador Civil. Habana 26 octubre-1868. BN. Mss 20138.
} 
medades venéreas y el control de las prostitutas 4 . No sabemos si por dejadez, ante la grave situación por la que atravesaba el país tras la guerra, o por que existían ideas antirreglamentistas entre las nuevas autoridades, la Sección de Higiene fue disuelta y las antiguas enfernas de la Quinta de la Higiene expulsadas. Sin embargo, unos meses después de haber acabado con el sistema entró de nuevo en vigor, debido al creciente número de enfermedades venéreas en el cjército de ocupación por lo que las autoridades norteamericanas se vieron obligadas a reorganizar y restablecer el sistema reglamentarista el siguiente años. La nueva organización del denominado servicio de Higiene Especial dependió el primer año de los Gobiernos Civiles y posteriormente de los Ayuntamientos ${ }^{6}$. Una orden civil ( $n^{\circ} 55$ del 27 de febrero de 1902) estableció que su administración y gobierno pasara a una Comisión Especial, supervisada por la Secretaría de Estado y Gobernación y compuesta por tres médicos, un abogado y un concejal. Uno de los tres médicos sería el presidente de la misma por votación entre los miembros que la componían y formaría parte de la Junta de Sanidad del Estado. ${ }^{7}$ El servicio de Higiene Especial no recibiría emolumentos alguno del estado, provincia o municipio, teniendo que subsistir de sus propios recursos, o sea de las que utilicen los servicios, las prostitutas.

Según las nuevas órdenes podemos detectar una cierta continuidad en el sistema. Se razonó la implantación de la nueva reglamentación, al igual que en el caso español, por el crecido número de enfermos venéreos entre la tropa y se pretendió que no costara un solo peso al estado. Hemos de recordar que la imposición de un impuesto, que según algunos era demasiado gravoso, para la autofinanciación del sistema fue una de las principales denuncias de las prostitutas y de los críticos al dominio español que consideraban que se estaba explotando "indecorosamente" a estas mujeres.

¿Pero hasta qué punto era necesaria la reglamentación? Para el Dr. Eugenio Molinet, del que sabemos que fue el primer Jefe Médico de la restablecida Sección Médica de la Higiene Especial tras la salida de los españoles de Cuba, la prostitu-

" GULLÓN, Alberto: "La prostitución reglada en La Habana de fines del siglo XIX.". En VẢZQUEZ, Francisco (coord.): "Mal Menor". Politicas y representaciones de la prostitución (siglos XVI-XLX). Cádiz, 1998.

"La causa principal por las que se establece la reglamentación es la misma por la que se estableció en 1873, el elevado número de enfermos venéreos que existian en las tropas. Para el caso español se llegó a estimar que existia un $10 \%$ de hombres infectados.

- "Artículo $1^{\circ}$. Organizado en esta Capital el servicio de Higiene Especial de la prostitución, cuyo objeto principal es evitar la propagación de la enfermedades venéreas y siffliticas, será regido como Jefe Superior del mismo, por el Alcalde Municipal, quien resolverá en última instancia las reclamaciones". Bases del servicio de Higiene Especial. En el Reglamento para el régimen de la prostitución en la ciudad de la Habana. Imprenta del "Avisador Comercial", de M. Pulido. Habana, 1899.

'Reglamento General para el servicio de la Higiene de la Prostitución o Higiene Especial de la Isla de Cuba. Imprenta y papelería "La Universal" de Ruiz y Hermano. Habana, 1902 
ción no era "...un mal necesario e irremediable como se sostiene por algunos; porque sin ella la sociedad podría existir y existir mejor; pero como dice Reus, constituye un estado inherente a nuestra manera de ser actual...." En este caso y a diferencia de alguno de sus colegas del XIX, Molinet, pone en duda una de las máximas del anterior siglo "el mal necesario", sin embargo, alega que era una constante en su sociedad y por lo tanto había que reglamentarla para evitar el contagio de enfermedades. Frente a los que abogan por la abolición de la reglamentación, el Dr. acude a un aparato bibliográfico-histórico reglamentarista, desde el tiempo de los romanos, y a datos de médicos coetáneos de otros países en donde el clandestinaje y las enfermedades venéreas se habían incrementado considerablemente. Por ello, Molinet, ante la auto-pregunta de si debe o no de estar reglada la prostitución se responde afirmativamente, pero debiendo tener más contenido sanitario que policial y, este último, "debe ser lo más suave posible."

Según el Dr. Molinet las medidas para regular la reglamentación deberían ir dirigidas a tratar de evitar que la meretriz enferme y que la prostituta enferma no contanine a los demás. Por ello, establece una serie de propuestas amplias, fruto de su experiencia y de los problemas de aplicación del reglamento vigente en 1899. Estas medidas pasan por un control higiénico adecuado de las meretrices y las "Casas"; una mayor revisión médica y más estricta, concentrado en el dispensario el peso de las citas sanitarias ${ }^{10}$; una apuesta muy atrevida, aunque no original, que pasaría por un cierto control del usuario masculino" y potenciar los estudios en venereología con el objetivo de hacer médicos entendidos en esa

${ }^{8}$ MOLINET, Eugenio: Memoria informe de la Sección Médica de la Higiene Especial... Opus cit. Pág 79

${ }^{9}$ Es necesario que exista una relación de pupilas con número de baños y habitaciones; existirán bidet con soluciones antisépticas para que se laven las mujeres y los hombres; debe exigirse que haya en cada habitación una buena cantidad de preservativos de goma, "pues son según la frase feliz de un venereólogo una tela de araña para el placer y una muralla para el contagio"; en lugar visible tener instrucciones de cómo asearse y las conveniencias de usar estas prácticas anteriormente descritas; deben rechazar a los hombres que no se dejen revisar antes del contacto o aquellos que sospechen tenga algún tipo de enfermedad. Todas estas medidas se les debe exigir a las dueñas de las casas bajo pena de multa o cierre de la casa. Ibídem, pág 80 .

${ }^{14}$ Visitas en el Dispensario y nunca en casa de las meretrices, salvo en caso de enfermedad que le imposibilite su traslado, al menos dos veces por semana y se permita examinar incluso si se estima necesario a diario; el reconocimiento debe ser completo; perseguir a las meretrices que no aparezcan por el reconocimiento; se deben aplicar medidas extremas con en Italia donde a toda mujer que este de baja y se encuentre ejerciendo el oficio se le haga "reo de lesión personal" y que a toda dueña que se le encuentre en su casa con mujeres enfermas tres veces consecutivas se les quite la licencia para siempre. Toda meretriz enferma debe ser recluida en el Hospital hasta su cura, pero considerando que el hospital no es una cárcel y el tratamiento a estas mujeres debe ser el adecuado. Ibídem, págs. 81-85. "El Dr. plantea revisiones periódicas para las fuerzas armadas, como policías, guardia rural, etc.. Asimismo, pretendía establecer una serie de consultas gratuitas para revisar a todos aquellos hombres que no tuviesen poder adquisitivo y con un horario lo suficientemente amplio para que pudiesen asistir sin problema a los dispensarios. Ibidem, pág 85. 
materia y exigirles el concurso' ${ }^{2}$. Estas ideas fueron en parte oídas por las autoridades ya que en el Reglamento Especial para el régimen de la prostitución en la Habana de 1902 se encuentran recogidas algunas de las propuestas del doctor ${ }^{13}$

\section{El régimen médico}

En enero de 1899 se restableció la Sección con un régimen nuevo en el servicio médico, cuyo objetivo era evitar "las enfermedades contagiosas por medio del contacto venéreo"; sin embargo, todos los servicios funcionaban conforme a disposiciones similares a las vigentes en el antiguo régimen ${ }^{14}$.

El jefe médico era director y médico de visita en la Quinta. Tenía seis médicos inspectores que reconocían determinadas zonas de la ciudad de La Habana dadas por el Director y debían hacer dos visitas médicas de inspección -una de ellas con un reconocimiento profundo en donde se empleaba el especulum y la otra un simple examen externo-; en caso de enfermedad la meretriz la enviaban a la Quinta y la daban de baja. También se practicaban tres veces a la semana reconocimientos a las meretrices denominadas ambulantes o a aquellas mandadas por algún motivo por la administración en un local anexo al que ocupaba la parte administrativa de la Sección ${ }^{15}$.

Pero a pesar de las medidas estipuladas, según Molinet, habría que dudar del sistema del control sanitario. Las prostitutas se avisaban "a voz en grito" cuando el médico entraba en las calles; una vez alertadas, muchas de las reglamentadas desaparecían temporalmente y las no regladas se escondían, lo que suponía una falta indudable de control, amén del "bochorno del decoro profesional" que suponía para los médicos.

\footnotetext{
${ }^{12}$ El profesor Fournier llama la atención especial sobre ese tema y Kaposi propone que en las facultades de medicina se creasen clínicas de venereologia, bajo el mismo pié que las médicas o quírúrgicas. Ibidem, pág. 86.

"Como en las condiciones adecuadas para dar la licencia a un burdel (art. 15); evitar el contacto con hombres sospechosos de enfermedad (art. 19); basar buena parte del servicio sanitario en un mayor control en el Dispensario, con ciertos días para las o los que no puedan pagar (arts. 36,37,38); concurso de los médicos (art. 33); etc...

${ }^{14}$ Reglamento para el régimen de la prostitución en la Habana. Imprenta del "Avisador Comercial" de M. Pulido. 1899. Capitulo. V. GULLÓN, Alberto: "E1 Hospital de la Sección de Higiene Especial en La Habana. El control médico de la prostitución. Siglo XIX." En el III Congreso Internacional de ADHILAC. Pontevedra, 2001 (en prensa).

1" Reglamento para el régimen de la prostitución en la Habana. Imprenta del "Avisador Comercial" de M. Pulido. 1899. Capítulo. V.
} 
En otras ocasiones el médico tenía que esperar a que las meretrices terminasen los servicios e incluso intentaban sobornarlos con dinero o prestaciones de alguna de las pupilas, lo que por los médicos se entendía como un "menoscabo de su prestigio profesional"; además, sufrían amenazas verbales continuas por parte de las prostitutas e incluso hubo quien tuvo que rechazar la agresión de algún proxeneta ${ }^{15}$.

También observamos que los exámenes a estas mujeres eran incompletos debido a que los reconocimientos no se hacían en sitios adecuados y a las astucias de las prostitutas 17 ; incluso era posible la transmisión de enfermedades al tener el médico que utilizar los mismos instrumentos quirúrgicos en sus revisiones.

Estas situaciones, que evidentemente ponen en entre dicho la efectividad de las normativas, van a tratar de corregirse en el reglamento de 1902, incrementándose el número de reconocimientos y horarios del dispensario; tratando de verificar las enfermedades de las que tenían que ir al dispensario; acompañando a los inspectores médicos la policía de higiene, etc... Medidas, que en su mayoría estaban reflejadas en el anterior reglamento y de las que dudamos, al menos en algunos casos, de su efectividad. ${ }^{18}$

Resulta importante reseñar la vital importancia que toma en este período el Dispensario ${ }^{19}$, que, aunque ya se instauró en la anterior etapa, ahora se transforma en la piedra angular del reconocimiento médico. Primero se solicitó al Gobemador Civil Federico Mora y al cambiar el servicio al control municipal se apoyo sin condiciones la propuesta.

En el dispensario se dividian a las mujeres en tres grupos de reconocimientos que se practicaban de 12 a 4 p.m. A las meretrices enfermas se les expedía una baja para ir a la Quinta de la Higiene; la policía las recogía y la lleva a la Quinta en ambulancia sin que las vieran, a no ser que hubiese una dueña que se com-

\footnotetext{
${ }^{16}$ MOLINET, Eugenio: Memoria informe de la Sección Médica de la Higiene Especial... Opus cit. pág 8.

" "... ocultando sus ulceraciones cubriéndolas con polvos de dientes húmedos, col-crean, tela fina de vejiga, polvos de carbón.. o bien hicieran desaparecer de su vagina, cuelfo o fondo de saco toda traza de supuración por modo de lavados abundantes." Las dadas de baja se marchaban a sus casas o se escondian antes de ir al hospital donde debian ser atendidas. Ibídem, pág. 9 .

${ }^{18}$ Reglamento General para el servicio de la Higiene de la Prostitución o Higiene Especial de la Isla de Cuba. Imprenta y papelería "La Universal" de Ruiz y Hermano. Habana, 1902, (art. 36-38).

19 "El Dispensario Médico de Higiene Especial está situado junto con la Sección Administrativa en el ala izquierda que en la calle de San Isidoro ocupa la Jefatura de Palicía. El Dispensario consta de una sala de espera, dos habitaciones destinadas a reconocimiento con un pequeño anexo para bidet, una sala para la oficina y una habitación para la dirección...." GULLON, Alberto: "E1 Hospital de la Sección de Higiene Especial en La Habana... Opus cit.
} 
prometiese a llevarla. Existía también un control de las que no pasaban las inspección el día señalado, visitándolas el médico en su casa y constatando su posible enfermedad. En caso de falta sin justificación se buscaban a las mujeres y se les obligaba a ir al día siguiente multándolas con un peso. Al final del día se justificaba por el Jefe Médico el total de mujeres reconocidas de ordinario y aquellas que en turno extraordinario, clandestinas, enfermas, etc., hubiesen pasado por el dispensario ${ }^{20}$. Parece que el sistema funcionó sanitariamente ya que era mucho más dificil utilizar argucias para ocultar las enfermedades venéreas ante las condiciones que existían en el dispensario y se evitaba que las meretrices escaparan del reconocimiento y del hospital en caso de estar enfermas.

En el Hospital de prostitutas venéreas u Hospital de San Antonio, establecido en 1873, se van a proponer algunas reformas por estas fechas que pretendían mejorar la situación del establecimiento. Frente a las consabidas obras, que todos los directores proponían para la mejora fisica del edificio ${ }^{21}$, es interesante observar la preocupación médica por una mejor forrnación e información de los doctores asistentes por lo que se formó una biblioteca exclusivamente de afecciones venéreas, ginecologia y prostitución. El instrumental quirúrgico detectado era bastante completo y moderno; además, el establecimiento tenía una sala de operaciones dotada de una autoclave, donde se realizan todo tipo de intervenciones ginecológicas y las mujeres allí recluidas podían ser asistidas u operadas por el médico que desearan, aunque no pertenecieran al servicio. Este aperturismo también se reflejó en la posibilidad de asistir a mujeres enfermas de males venéreos que no fueran prostitutas y en el régimen interno establecido, quitándole el aspecto "carcelario que tenía" y haciendo compatible las necesidades hospitalarias con la obligada reclusión hasta la sanidad venérea."

Similar a lo que ocurría en épocas anteriores existía el problema del tedio y del aburrimiento de las recluidas en el Hospital, ya que "la mayoría padecen de enfermedades que no les obliga a guardar cama" por lo que surgían problemas de irritabilidad entre las enfermas, juegos que denominan estúpidos, etc...., que alteraban hasta cierto punto el orden del establecimiento. Por ello los directores se plantearon, siguiendo los modelos del Dr. D. Claudio Delgado ${ }^{23}$, el establecimiento de unas medidas dirigidas a entretener y, al mismo tiempo, tratar de ayu-

\footnotetext{
${ }^{20}$ Reglamento para el régimen de la prostitución en la Habana. Imprenta del "Avisador Comercial" de M. Pulido. 1899. Reglamento General para el servicio de la Higiene de la Prostitución o Higiene Especial de la Isla de Cuba. Imprenta y papelería "La Universal" de Ruiz y Hermano. Habana, 1902. "GLLLÓN, Adberto: "El Hospital de la Sección đe Higiene Especial en La Habana... Opus cit.

${ }^{22}$ MOLINET, Eugenio: Memoria informe de la Sección Médica de la Higiene Especial ... Opus cit, pág 63.

${ }^{23}$ MARTÍNEZ FORTUN, Otelio: Estudio blográfico del médico español D. Claudio Delgado y stt aportación de la fiebre amarilla. Madrid, 1954.
} 
dar a estas mujeres. Para ello, se establecieron una serie de ejercicios mentales y físicos con el objetivo de tener entretenidas a las asiladas, proponiéndose labores de aguja, lectura en voz alta y una sección de música ${ }^{24}$. Estas tibias medidas, comparadas con la etapa anterior donde se llegó a pretender un mejoramiento físico y moral dirigido a la recuperación de la autoestima como paso inicial para poder salir de la prostitución, se nos antojan convencionales y enmarcadas dentro del concepto puramente sanitario, acercándonos al limitado objetivo de la reglamentación-prevenir los atentados contra la moral pública y a garantizar la salud de las colectividades-, pero sin ofertar una minima oportunidad de "regeneración" a estas mujeres, más allá de la posibilidad de una renuncia avalada por su comportamiento y algunas personas que lo ratifiquen.

Sin embargo, los escritos médicos nos plantean la redención de las prostitutas, afirmando que no pasaba por la prohibición ya que no daba resultados, sino por poner a la mujer en condición de subsistir por si misma sin necesidad de apelar a estos recursos y facilitándole el medio para que no recaiga en el viejo oficio; a pesar de ello, las medidas detectadas en el hospital, que en otros tiempos tuvieron ese objetivo, no iban ahora dirigidas en esa dirección.

Ello nos lleva a pensar en una falta de interés real por tratar de ofertar a estas mujeres una posibilidad en el campo laboral, triunfando la idea, avalada por un conjunto de intereses públicos y privados, que consideraba a la prostitución como un mal necesario dentro de la moralidad burguesa dominante que se sentía incapaz de acabar con el fenómeno prostibulario.

\section{¿Prostitutas?}

Existen muchas definiciones sobre prostitución para la época, pero de las que hemos encontrado la más ilustrativas es la realizada por el Dr. Ramón María Alfonso: "La prostitución es el comercio bi-sexual, mediante precio. Esto constituye su carácter diferencial, privativo, único"2s. El Dr. Alfonso parece que rompe con la idea de algunos predecesores que acusaban a una mujer de prostituta por

\footnotetext{
${ }^{24}$ "La lectura en alta voz y en común seria de gran provecho, con una buena elección de las materias es seguro que durante una hora al día por lo menos, se mantendría ocupada la atención de todas las enfermas, obteniéndose un efecto saludable en muchas de ellas. La música bien elegida sería un verdadero y agradable remedio para muchas de las enfermas y para todas un excelente entretenimiento por espacio de una o dos horas diarias, sobre todo en el espacio de tiempo que media entre la comida y la hora del descanso para dormir", MOLINER, Eugenio: Memoria informe de la Sección Médica de la Higiene Especial... Opus cit, pág. 66.

${ }^{25}$ ALFONSO, Ramón $M^{3}$ : La reglamentación de la prostitución. Breves apuntes sobre como debe ser en Cuba. Habana, 1912. Pág 48-49.
} 
el simple hecho de efectuar lo que denomina "actos de provocación" entre los que se encontraban las miradas o palabras a los transeúntes, recibir hombres en salas, asistir a los bailes y libaciones con los amigos, penetrar con visitas en el interior de la vivienda, ir en paños menores al baño si hay que salir de la vivienda, recorren los cuartos sin hacer caso de lo que se pueda ver; sentarse o parar en el balcón enseñando más de lo permitido por el decoro, proferir palabras o cantos obscenos, recorrer las calles o plaza acentuando las curvas de su cuerpo, etc... ; en fin, un conjunto de actos que nos identifica cuales son los comportamientos prescritos o cercanos a lo que se entiende hacen las prostitutas y que las definen como tales. El Dr. asume estos comportamientos como rasgo de identificación, aunque en este caso, y con la nueva ley, afirme que "todo ello es una prueba de incivilidad, de indecencia e inmoralidad, en una palabra, pero no es prueba de prostitución" ${ }^{126}$, de ahí la justificación para el crecinniento de las denominadas meretrices clandestinas.

Muchos de los elementos expuestos anteriormente como comportamientos indecorosos fueron razones que se esgrimieron en el anterior período como entrada en el mundo de la prostitución cubana y similares a las dadas en otros países ${ }^{27}$. Sin embargo, en este período se pone de manifiesto un nuevo sistema de captación de prostitutas a tener en consideración, el denominado "trata de blanca" que hizo que por esta época saltaran las alarmas de la sociedad, "aquellas desgraciadas que eran engañadas y raptadas hacia otros países" y aunque estamos seguros de algún caso para Cuba, según los informes médicos, no era lo usual. En parte, porque existía una reglamentación que obligaba a estar estas mujeres con médicos o agentes de la autoridad a los que hubieran podido informar y, por otra, que el modus operandi de los reclutadores era muy distinto ${ }^{28}$. Los denominados "agentes provocadores" existían, pero de los que iban al extranjero, especialmente a Francia, eran pocos y las mujeres que traían parece ser que estaban ya forjadas en lupanares de sus países ${ }^{29}$.

\footnotetext{
"ALFONSO, Ramón M": La reglamentación de la prostitución. Breves aptintes... Opus cit, pág 49.

${ }^{27}$ ANDREO, Juan y GULLÓN, Alberto: "Vida y muerte de la mulata. Crónica ilustrada de la prostitución en Cuba del XIX. En Anuario de Esiudios Americanos, LIV-., Sevilla, 1997.

28 "... pues ni es Europa, por lo general, la que nos surte directamente del $<$ articulo $\gg$ en cuestión, ni existe en esta capital casa dedicada al secuestro de las extranjeras, ni nuestras leyes de inmigración favorece ese indigno tráfico." ALFONSO, Ramón $\mathrm{M}^{\mathrm{n}}$ : La reglamentación de la prositución. Breves apuntes... Opus cit, 17.

2* "Por lo regular, ninguna extranjera viene enganada; la joven inınigrante sabe a lo que viene y lo hace gustosa por el atán de lucro. Y lo saben sus padres, si existen, que por un anticipo de 200 a 300 francos, la confian al mercader o celestina que las contrata, pensando que esto les produce más a todos en dos meses, que lo que gana la chiquilla en seis, haciendo penosamente el $\ll$ trottoir $>$ con San Lázaro por toda perspectiva." ALFONSO, Ramón Ma: La reglamentación de la prosititución. Breves apuntes... Opus cit, pág. 18. Ello pone en evidencia la venta de seguranente niñas, por parte de los padres ante las necesidades y el elevado número de hijos, venta similar a la que se produce en la actualidad y se denuncian en algunos países.
} 
Parece ser que la mayor parte de las prostitutas extranjeras procedían, con excepción de las españolas, del arco caribeño, lo que evidencia que se mantienen las mismas redes que existían en la época colonial. Estas mujeres, en su mayoría, habían trabajado con anterioridad los burdeles de Méjico, Estados Unidos, Panamá y Venezuela, llegando de Europa bajo la apariencia de artistas, bailarinas, floristas, mecanógrafas, etc... Son la dueñas de las casa de lenocinio las que controlan el tráfico de estas mujeres, intercambiándolas en el arco caribeño. Utilizan para ello a agentes reclutadores, que no necesariamente son profesionales, en tránsito por esos países que hablan sobre las "bondades" de los burdeles para los que trabaja y lo mucho que ganan sus pupilas; además no existía ninguna ley, excepto para las menores, que les prohibiera a las prostitutas reconocidas, mayores de edad, libre y voluntariamente ir a $\mathrm{Cuba}^{30}$. Para controlar la entrada, al menos de las menores, se sugirió en un informe de la Comisión de Higiene Especial en 1909 que se pusiera un funcionario de policía en la casilla de Inspección de Pasajeros que conociera al personal de agentes de prostíbulos, "casi todos <<apaches $>$ conocidos en la ciudad para la detención de los sospechosos"3l, aunque no sabemos nada sobre su posible eficacia.

Por los datos recogidos desde el fin de la guerra hasta 1912, el número de meretrices osciló entre quinientas y seiscientas, unas cien más que en la época colonia; sin embargo, abría que exceptuar los primeros años que, por las causas de la guerra y las necesidades posteriores, hizo que se incrementase la cifra hasta las 850 , aunque disminuyó rápidamente. Pero estas son las estimaciones de las prostitutas regladas (las dependientes, las independientes y las ambulantes ${ }^{32}$ ), frente a ellas estaban las clandestinas que según informadores de la época multiplicarían el número real por diez.

En el mundo médico existía una clara preocupación por estas últimas, consideradas como un "gen cancerigeno de la sociedad, que infecta con su mal venéreo y que no se somete a las directivas del reglamento, por ello debe ser perseguida e inscrita". Según el Dr Ramón Ma no existía un solo modelo o tipo de clandestina y jugando con los prototipos de imágenes, los prejuicios sociales y los comportamientos que se definían como actos de prostitución establece una clasi-

\footnotetext{
so “..El texto de la ley se refiere únicamente a las prostitutas importadas para ejercer como tales: «The importation into Cuba of woman for the purpose of prostitution is forbidden $>\mathrm{y}$ si en las cámaras de $1^{\text {a }}$ de un vapor viene una mujer extranjera, no asiática, sana, mayor de edad, que viaja por su libre albedrio y exhibe recursos monetarios que acrediten su solvencia, aunque declare ser meretriz, no hay fundamentos legales para que el Departamento de Inmigración le prohíba su libre acceso. ALFONSO, Ramón M: La reglamentación de la prostitución. Breves apuntes... Opus cit, pág 23.

". Si se demostraba la infracción debía ser castigado, aplicándole las penas prescritas en la Orden Civil 155 con prisión que no excedía de cinco años a multa de cinco mil pesos. Ibídem.

32 GULLON, Alberto: "Un acercamiento a la prostitución cubana de fines del siglo XIX". En La Nación Soñada: Cuba. Puerto Rico y Filipinas ante el 98 . Aranjuez 1996, 497-508 págs.
} 
ficación. En primer lugar coloca a las denominadas "fleteras" que serían prostitutas de color de más ínfimo nivel. Después sitúa a las clandestinas propiamente dichas, casi todas blancas y menores de edad, que vestían igual que las mujeres denominadas honradas y se distinguían por ir solas con una compañera o un niño de pocos años; posiblemente estas ejercían la prostitución ocasionalmente y para nada llevarian una forma de vida marginal, mas bien aportarian un sueldo que entraría en la familia con conocimiento de esta. Otro grupo de clandestinas sería la que disponen de un domicilio particular de apariencia más o menos lujosa, que de tarde en tarde aparece en las casas de citas, "pagado por varias personas acaudaladas que funcionan como protectores con horas establecidas." Y por último, introduce a un grupo de mujeres con oficios, volvemos a ciertos prejuicios de la sociedad patriarcal cubana que no veía con buenos ojos que trabajasen las mujeres de cara al público, compuestas por artistas, mecanógrafas, etc... que cobrarían sus servicios por un traje de valor, unas joyas o un abono de temporada de teatro. ${ }^{33}$

El problema para el control de estas mujeres que estaban fuera de los reglamentos consistía en cómo demostrar que ejercía el oficio. Así, el Dr. Alfonso denunciaba la falta de leyes que persiguiesen efectivamente a estas mujeres, ya que las denuncias de los vecinos eran anónimas o cuando se presentaba el denunciador las molestias por el pleito e incluso las amenazas personales hacian que estos hechos se oscureciesen. Incluso a la hora de <<instruir el expediente de clandestinaje $>>$ había que buscar la confirmación de los vecinos, que por temor no contestaban al haber visto a alguien influyente o ser amenazados fisicamente. Se percibe, además, un círculo de sociabilidad (bodegueros, arrendadores, tenderos, etc...) que obtienen algún beneficio del trabajo de estas mujeres y que las encubren haciendo declaraciones sobre la honradez y la "aceptable moralidad" de estas; amén de los posibles protectores que en ocasiones son fuertes personajes y de la ineficacia policial por la falta de cualificación o porque se dejaban sobornar.

Un cambio importante que detectamos con respecto a la etapa anterior es la denominada Zona de Tolerancia ${ }^{34}$, cuyo objetivo era aislar y esconder el fenómeno prostibulario que se consideraba por los grupos sociales dominantes como una lacra social aunque necesaria. En la etapa colonial las meretrices vivían diseminadas por distintos puntos de la ciudad ( áreas portuarias y comerciales, caminos de salida y entrada de la ciudad, etc...) y aunque las casas de lenocinio tenían asignados puntos concretos en determinadas calles y unas duras reglas de control por parte de las autoridades, para algunos suponía un "escánđalo perpetuo"335. Con la

\footnotetext{
"ALFONSO, Ramón $\mathrm{M}^{\text {": }}$ La reglanentación de la prostitución. Breves apuntes... Opus cit, pág 47. is "La zona de tolerancia es un barrio o sector de la población, casi siempre en perímetro urbano, o en aquellos sitios donde el tránsito público es menor, que se señala como domicilio forzoso a las meretrices reglamentadas, para el ejercicio de su industria". Ibídem.
} 
llegada de los norteamericanos la idea de establecer una zona específica donde desarrollar estas actividades triunfó frente a los que opinaban que esta medida se asemejaba a un sistema carcelario, justifícándose en que "la prostitución es una industria insalubre y que puede causar perjuicios a la moral y al orden, industria insalubre y peligrosa que si no es indispensable es por lo menos, hoy por hoy, necesaria"366; asi, las prostitutas se van a circunscribir a una zona tolerada por las autoridades o a unas casas autorizadas fuera de la zona ${ }^{37}$. La idea de los reglamentaristas pasaba por concentrar a estas mujeres en un espacio para tenerlas mejor controladas sanitariamente y poder perseguir a las clandestinas; al mismo tiempo, limitaban, según ellos, que cundiera el mal ejemplo que podían dar estas mujeres $y$, por lo tanto, la mejor solución era recluirlas en zonas apartadas de la ciudad.

Pero a medida que va avanzando el siglo observamos que la efectividad de la zona era limitada, máxime cuando informes como los del Dr. Ramón $\mathrm{M}^{\mathbf{a}}$ afirman que tras promulgarse las nuevas leyes y consignarse en ella derechos civiles y políticos, sin distinción de sexo, "no se concibe la continuación ni la utilidad de las zonas" al caer en contradicción con la libertad del individuo y, por lo tanto, sólo ejercían en la zona aquellas mujeres "más disciplinadas"; así, se explicaría el incremento de las denominadas clandestinas que ocupaban prácticamente toda la ciudad, $\sin$ que se le pudiese obligar a residir en la zona, puesto que la Constitución y el derecho de gentes lo prohibía.

Otro de los problemas que encontramos sobre la zona y que se puso de manifiesto cuando se intentó trasladarla de sítio fue el de la jurisdicción. En teoría la designación de la zona respondía a los ayuntamientos, pero había que contar con las ideas de los alcaldes, corporaciones municipales y las autoridades sanitarias.

Se percibe un juego de estrategias, que tiene que ver con el crecimiento de la ciudad y el interés que suscita para determinados grupos el ocupar las anteriores zonas apartadas que ahora aparecen como sitios de tránsito o centros industriales. Asimismo, se puede observar como vecinos influyentes presionan para que la zona se traslade a propiedades que tienen en las afueras y que pretenden se urbanicen, ya que “... no solo se erigirán allí casas, fabricadas por él o por otros, pues hay inquilinos seguros desde ese momento, sino que habrá que dotar a esa

\footnotetext{
${ }^{35}$ GULLÓN, Alberto J: "Poder y marginación en La Habana a fines del período colonial". En Gades, $\mathrm{n}^{0}$ 23, Cádiz, 1999

${ }^{36}$ MOLINET, Eugenio: Memoria informe de la Sección Médica de la Higiene Especial... Opus cit, pág 88.

${ }^{37}$ Existían burdeles fuera de la zona de tolerancia, especialmente en el centro de la ciudad, pero tenían que tener el beneplácito de los vecinos cercanos; además debían cumplir unas estrictas normas, fruto de la experiencia acumulada de antiguos reglamentos, como estar en calles solitarias y, si no lo eran, en plantas altas y con mamparas para que no se viesen los interiores; había unos horarios de limpieza, de siete a diez de la mañana; no podian pintar los locales con colores llamativos, etc...
} 
parcela de terreno de agua, alumbrado calles, aceras, policías, etc...". Otras veces es el conjunto de intereses de aquellos que viven del barrio existente -bodegueros, tenderos, arrendadores, etc..- que aducen todo tipo de razones para que la zona no se mueva y son tan satisfactorias que a veces revocan acuerdos tomados. También se puede observar presiones sobrc el jefe de Higiene para trasladar la zona a sitios que, a juicio de los médicos, son inadecuados por lo que las meretrices emigran y el servicio se desbarata ${ }^{38}$.

En general intereses o presiones sobre las autoridades que podian tener algún tipo de jurisdicción o influencias en las medidas a tomar sobre la zona que para nada contemplaban los intereses o las opiniones de las afectadas, aunque estas reaccionasen casi inmediatamente con un incremento del clandestinaje.

A modo de conclusión podríamos decir que en general se mantuvo una cierta continuidad, a pesar de que en un momento inicial se tratara de hacer desaparecer la reglamentación; incluso las razones que se esgrinieron para el mantenimiento de la Sección de Higiene fueron similares a las esgrimidas por los españoles en su momento, como el incremento de número de enfermos venéreos entre las tropas allí destinadas.

En el aspecto sanitario la acción sobre estas mujeres estuvo orientada al control de las enfermedades venéreas y dirigida por médicos, si bien el dispensario será ahora la piedra angular del reconocimiento y control de éstas; asimismo, observamos en el hospital un alejamiento de las medidas "regenerativas" sobre estas mujeres, centrando su actividad casi en exclusividad en la recuperación sanitaria de las enfermas.

Los fundamentos o razones para la identificación de las prostitutas fueron similares, si bien la aparición de una nueva ley-donde los derechos individuales estaban plasmados en igualdad de condiciones con respecto a otros ciudadanoscomplicó la detención de estas mujeres, incrementándose el clandestinaje.

La diferencia más notable que hemos encontrado con respecto a la etapa anterior ha sido la zona de tolerancia. Frente a la diseminación de los prostíbulos, se marca una zona concreta en la ciudad, a ser posible alejada de las zonas más concurridas, en donde se permite el establecimiento de las casas, aunque existen algunas excepciones. Con ello, los reglamentistas pretendían un mayor control sanitario y de movimiento de estas mujeres, aunque por los datos que tenemos parece que no obtuvo el éxito esperado.

${ }^{38}$ ALFONSO, Ramón $\mathrm{M}^{\mathrm{a}}$ : La reglamentación de la prostitución. Breves apuntes..... Opus cit, pág.114. 